\title{
Performance Modeling of an Experimental Laser Propelled Lightcraft
}

\author{
Ten-See Wang ${ }^{*}$ \\ NASA Marshall Space Flight Center, Huntsville, AL 35812 \\ Yen-Sen Chen ${ }^{\dagger}$ and Jiwen $\mathrm{Liu}^{\ddagger}$ \\ Engineering Sciences, Inc., Huntsville, AL 35802 \\ Leik N. Myrabo $\$$ \\ Rensselaer Polytechnic Institute, Troy, NY 12180 \\ and \\ Franklin B. Mead, Jr. ${ }^{* *}$ \\ Air Force Research laboratory, Edwards AFB, CA 93524
}

\begin{abstract}
$\underline{\text { Abstract }}$
A computational plasma aerodynamics model is developed to study the performance of an experimental laser propelled lightcraft. The computational methodology is based on a timeaccurate, three-dimensional, finite-difference, chemically reacting, unstructured grid, pressurebased formulation. The underlying physics are added and tested systematically using a buildingblock approach. The physics modeled include non-equilibrium thermodynamics, nonequilibrium air-plasma finite-rate kinetics, specular ray tracing, laser beam energy absorption and refraction by plasma, non-equilibrium plasma radiation, and plasma resonance. A series of transient computations are performed at several laser pulse energy levels and the simulated physics are discussed and compared with those of tests and literatures. The predicted coupling coefficients for the lightcraft compared reasonably well with those of tests conducted on a pendulum apparatus.
\end{abstract}

\footnotetext{
Team Lead, Senior Member AIAA

' President, Member AlAA

- Senior Research Engineer, Member AIAA

- Associate Professor, member AIAA

Sr. Scientist, Member AIAA
} 


\section{$\underline{\text { Nomenclature }}$}

$A_{j} \quad$ reaction rate constant of the $j$-th reaction

$A_{p}, A_{m}$ matrix coefficients of transport equations

c speed of light in a vacuum.

$D \quad$ species diffusivity

$e_{v} \quad$ vibrational energy

$e_{v}^{e q} \quad$ equilibrium vibrational energy

$\vec{F} \quad$ flux vector

$\mathrm{g}_{i} \quad$ Gibbs' free energy of species $\mathrm{i}$

$H \quad$ total enthalpy

I intensity

$J \quad$ emission coefficient

$k_{b} \quad$ Boltzmann's constant

$k_{\mathrm{c}} \quad$ Coulomb constant

$k_{v} \quad$ thermal conductivity of the vibrational energy equation

$M_{w} \quad$ molecular weight

$\mathrm{m}_{\mathrm{e}} \quad$ electron mass

$N_{i} \quad$ quantum level population

$n \quad$ refraction index

$\vec{n} \quad$ unit normal vector

$n_{e} \quad e$ lectron number density

$P_{r} \quad$ Prantdl number 


$$
\begin{array}{ll}
p & \text { gas static pressure } \\
p_{e} & \text { partial electron temperature } \\
Q_{e r} & \text { elastic collision energy transfer } \\
Q_{r} & \text { net radiative heat flux } \\
\mathrm{q}_{\mathrm{e}} & \text { electron charge } \\
R & \text { gas constant } \\
\vec{r} & \text { position vector } \\
S & \text { source terms } \\
T & \text { gas temperature, } \mathrm{K} \\
T_{e} & \text { electron temperature, } \mathrm{K} \\
T_{t} & \text { translational temperature, } \mathrm{K} \\
T_{v} & \text { vibrational temperature, } \mathrm{K} \\
u_{i} & \text { velocity components } \\
V & \text { velocity magnitude } \\
x_{i} & \text { Cartesian coordinates } \\
& \\
& \\
& \\
&
\end{array}
$$

\section{Greek Symbols}

$\begin{array}{ll}\Delta t & \text { time step size } \\ \varepsilon_{0} & \text { permittivity } \\ \phi & \text { dependent variable } \\ \Gamma & \text { control surface } \\ \kappa 1 & \text { laser absorption coefficient. }\end{array}$




$\begin{array}{ll}\lambda^{\prime} & \text { electron species thermal conductivity } \\ \lambda_{i} & \text { laser wavelength } \\ \mu & \text { viscosity } \\ v_{i j} & \text { stoichiomatric coefficient } \\ \Omega & \text { control volume domain } \\ \dot{\omega}_{i} & \text { chemical reaction source term } \\ \omega_{\mathrm{p}} & \text { plasma frequency } \\ \rho & \text { density } \\ \sigma r & \text { Schmidt number } \\ \tau_{T} & \text { vibrational-translational relaxation time scale } \\ \tau_{i j} & \text { shear stress tensor }\end{array}$

\section{Introduction}

Beamed energy propulsion was first promoted by Kantrowitz ${ }^{\mathrm{K} 1}$. Since then, a propulsion system supported by a laser-sustained plasma has been the subject of many studies. ${ }^{\mathrm{Gl}, \mathrm{B} 2, \mathrm{Jl}, \mathrm{M} 4}$ The main advantage gained by laser propulsion is the low-weight system from decoupling the energy source from the vehicle, and high specific impulse from low fuel consumption. The first ground and flight tests of a ground-based laser propelled vehicle was reported in $1998,{ }^{\mathrm{M} 1}$ in which spinstabilized free flights with active tracking and beam control were accomplished to altitude of 4 $\mathrm{m}$. Six months later, the spin-stabilized vertical free flights reached $30 \mathrm{~m} .{ }^{\mathrm{M} 2}$ These axisymmetric vehicles are of a special design among many innovative concepts in which a nosecone shaped forebody, an annular shroud, and a parabolic afterbody are the only major components. Vehicles 
of such a design is hereby named as the "Laser Lightcraft" in this study. The layout of a computational grid for a Laser Lightcraft is shown in Fig. 1. The parabolic afterbody engine serves both as an aerospike nozzle and also the main receiving optic, whereas the annular shroud surrounds the ring focus of the parabolic optic. The Laser Lightcraft uses a Rocket-Based Combined Cycle (RBCC) to operate in both the rocket and air-breathing modes. When operating in an air-breathing mode, the specific impulse is infinity since no fuel is consumed. These successful tests ${ }^{\mathrm{M} 1, \mathrm{M} 2}$ demonstrated the feasibility of launching small payload with a Laser Lightcraft.

When focused beam energy strikes the shroud of a Laser Lightcraft, free electrons form to invoke Inverse Bremsstrahlung (IB) and the optical breakdown ensues. In order to achieve maximum intensity and to avoid plasma resonance, repetitive short pulses are used. As such, repetitive Pulsed Detonation Waves (PDW) are generated to propel the vehicle, hence the Laser Lightcraft engine is also know as a PDW engine. It can be seen that the underlying physics involved such as IB and PDW are so complicated that a simple system model is not capable of describing the phenomena, let alone predicting the thrust performance and the associated flow and thermal environments necessary for designing for the structure integrity. A detailed computational plasma aerodynamics model is therefore needed for the design and scaling of future Laser Lightcrafts.

From May to July, 1999, performance data in the form of coupling coefficients were taken in a series of indoor tests for the Model \#200 series vehicles. These test were performed under a cooperative effort among Rensselaer Polytechnic Institute, Air Force Research Laboratory and NASA Marshall Space Flight Center (MSFC), to enhance Laser Lightcraft propulsion research. 
These data are the first of a kind benchmark quality data for computational model development. Armed with these data for comparison purpose, a computational plasma aerodynamics methodology is developed as a first attempt to predicting the thrust performance of a PDW engine propelled Laser Lightcraft.

\section{Computational Plasma Aerodynamics Development}

Some attempts have been made to analytically or computationally model the physics inside continuous wave $(\mathrm{CW})$ devices. An early effort by Raizer ${ }^{\mathrm{R} 2}$ assumed constant-pressure onedimensional (1D) flow in air with heat addition by a laser. Thermal conduction was considered dominant and the radiation loss from plasma was ignored. Kemp and $\operatorname{Root}^{\mathrm{K} 2}$ later extended this 1D analysis to hydrogen and to include thermal radiation. Molvik, Choi, and Merkle ${ }^{\mathrm{M} 4}$ extended this problem to a two-dimensional (2D) structured-grid hydrogen flow and implemented real ray tracing, but ignored thermal radiation. Jeng and Keefer ${ }^{\mathrm{J} 1}$ did similar analysis and added thermal radiation. In this work, a multidimensional unstructured-grid computational plasma aerodynamics methodology is developed, with emphases on nonequilibrium effects and laserinduced physics such as laser - plasma interactions. Realistic laser absorption coefficients are used. The governing equations will be described first, with the auxiliary equations that computing the nonequilibrium and laser induced physics to follow.

\section{The Governing Equations}

To properly describe the plasma aerodynamics involved in laser propelled propulsion, the time-varying transport equations of continuity, species continuity, momentum, global energy 
(total enthalpy) and electron energy are formulated and written in a Cartesian tensor form:

$$
\begin{aligned}
& \frac{\partial \rho}{\partial t}+\frac{\partial}{\partial x_{j}}\left(\rho u_{j}\right)=0 \\
& \frac{\partial \rho Y_{i}}{\partial t}+\frac{\partial}{\partial x_{j}}\left(\rho u_{j} Y_{i}\right)=\frac{\partial}{\partial x_{j}}\left[(\rho D) \frac{\partial Y_{i}}{\partial x_{j}}\right]+\dot{\omega}_{i} \\
& \frac{\partial \rho u_{i}}{\partial t}+\frac{\partial}{\partial x_{j}}\left(\rho u_{j} u_{i}\right)=-\frac{\partial p}{\partial x_{i}}+\frac{\partial \tau_{i j}}{\partial x_{j}} \\
& \frac{\partial \rho H}{\partial t}+\frac{\partial}{\partial x_{j}}\left(\rho u_{j} H\right)=\frac{\partial p}{\partial t}+Q_{e c}+\frac{\partial}{\partial x_{j}}\left(\frac{\mu}{P_{r}} \nabla H\right)+\frac{\partial}{\partial x_{j}}\left(\left(1-\frac{\mu}{P_{r}}\right) \nabla\left(V^{2} / 2\right)\right) \\
& \frac{\partial}{\partial t}\left(\frac{2}{3} k_{b} n_{e} T_{e}\right)+\frac{\partial}{\partial x_{j}}\left(\frac{2}{3} k_{b} n_{e} T_{e} u_{j}\right)=\frac{\partial}{\partial x_{j}}\left(\lambda_{e} \frac{\partial T_{e}}{\partial x_{j}}\right)+Q_{r}-Q_{e c}
\end{aligned}
$$

where the shear stress $\tau_{i j}$ can be expressed as:

$$
\tau_{i j}=\mu\left(\frac{\partial u_{i}}{\partial x_{j}}+\frac{\partial u_{j}}{\partial x_{i}}-\frac{2}{3} \frac{\partial u_{k}}{\partial x_{k}} \delta_{i j}\right)
$$

The beam energy absorption, beam energy transfer, and plasma radiation are invoked through the source terms of the global energy and the electron energy equations, whereas the plasma species are generated through the source terms of the species continuity equations. 


\section{Numerical Scheme}

The cell-centered scheme is employed that the volume surfaces are represented by the grid cell surfaces. The transport equations can be written in integral form as:

$$
\frac{\partial}{\partial t} \int_{\Omega} \rho \phi d \Omega+\oint_{\Gamma} \vec{F} \cdot \vec{n} d \Gamma=\oint_{\Omega} S_{\phi} d \Omega
$$

where $\Omega$ is the domain of interest and $\Gamma$ denotes the surrounding surfaces; $\vec{n}$ is a unit normal

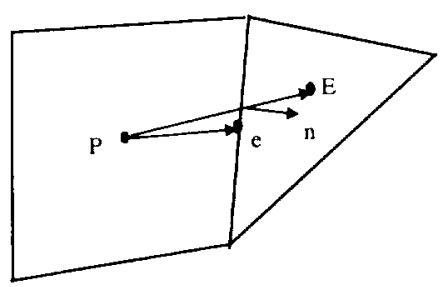

vector of $\Gamma$ in the outward direction. The flux function $\vec{F}$ contains the inviscid and the viscous flux vectors, And, $\phi_{E}-\phi_{P} \approx \overline{\nabla \phi}_{e} \bullet\left(\vec{r}_{E}-\vec{r}_{P}\right)$ where $\bar{\nabla} \phi_{e}$ is interpolated from the neighbor cells $E$ and $P$.

$$
\vec{F}=\rho \vec{V} \phi-\mu_{\phi} \nabla \phi
$$

For the face $e$ between control volumes $P$ and $E$, the diffusive flux can be approximated as:

$$
(\nabla \phi \bullet \vec{n})_{e} \approx \frac{\phi_{E}-\phi_{P}}{\left|\vec{r}_{E}-\vec{r}_{P}\right|}+\overline{\nabla \phi}_{e} \bullet\left(\vec{n}-\frac{\vec{r}_{E}-\vec{r}_{P}}{\left|\vec{r}_{E}-\vec{r}_{P}\right|}\right)
$$


The finite volume formulation of flux integral can be evaluated by the summation of the flux vectors over each face,

$$
\oint_{\Gamma} \vec{F} \cdot \vec{n} d \Gamma=\sum_{j=k(i)} F_{i, j} \Delta \Gamma_{j}
$$

where $k(i)$ is a list of faces of cell $i, F_{i, j}$ represents convection and diffusion fluxes through the interface between cell $i$ and $j$, and $\Delta \Gamma_{j}$ is the cell-face area.

The convective flux is evaluated through the upwind-cell quantity by a linear reconstruction procedure to achieve second order accuracy: $\phi_{e}=\phi_{u}+\phi_{e} \nabla \phi_{u} \bullet\left(\vec{r}_{e}-\vec{r}_{u}\right)$ where the subscript $u$ represents the upwind cell and $\psi_{e}$ is a limiter used to ensure that the reconstruction does not introduce local extrema. The limiter proposed by $\operatorname{Barth}^{\mathrm{B} 3}$ is used here. Defining $\phi_{\max }=\max \left(\phi_{u}, \phi_{j}\right), \phi_{\min }=\min \left(\phi_{u}, \phi_{j}\right)$ (and assuming $\phi_{e}^{0}$ is computed with $\left.\psi_{e}=1\right)$ the scalar $\psi_{e}$ associated with the gradient at cell $u$ due to edge $e$ is:

$$
\psi_{*}=\left\{\begin{array}{cc}
\min \left(1 \frac{\phi_{\max }-\phi_{u}}{\phi^{0}-\phi_{u}}\right) & \text { if } \phi^{0 \prime}-\phi>0 \\
\min \left(1, \frac{\phi_{\min }-\phi_{u}}{\phi^{0}-\phi_{u}}\right) & \text { if } \phi^{0}-\phi<0 \\
1 & \text { if } \phi^{0}-\phi=0
\end{array}\right.
$$

\section{Solution Procedures}

A general implicit discretized time-marching scheme for the transport equations can be written as below, 


$$
\left(\frac{\rho^{n}}{\Delta t}+A_{\rho}\right) \phi_{p}^{n+1}=\sum_{m=1}^{N B} A_{m} \phi_{m}^{n+1}+\frac{\left(\rho \phi_{p}\right)^{n}}{\Delta t}+S_{\varphi}
$$

where $N B$ means the neighbor cells of cell $\mathrm{P}$. The high order differencing term and cross diffusion term are treated using known quantities and retained in the source term and updated explicitly. The second term of the right-hand side (RHS) is a perturbation which comes from the RHS. A predictor/corrector solution algorithm is employed to provide coupling of the governing equations. The discretized finite-volume equations form a set of linear algebra equations, which are non-symmetric matrix system with arbitrary sparsity patterns. The preconditioned Bi$\operatorname{CGSTAB}^{\mathrm{F} 1}$ and GMRES $(m)^{\mathrm{S} 2}$ matrix solvers are used to efficiently solve the linear algebra equations.

\section{$\underline{\text { Auxiliary Equations }}$}

\section{Thermal Non-Equilibrium Energy Equations}

For high temperature flows, thermal non-equilibrium state may be important. In Landau and Teller's derivation, ${ }^{\mathrm{L} 1}$ a master equation is employed to describe the evolution of the population of quantum level $N_{i}$. This master equation is written as:

$$
\frac{d N_{i}}{d t}=N \sum_{j=0}^{I_{\max }} K_{j \rightarrow i} N_{j}-N \sum_{j=0}^{I_{\max }} K_{i \rightarrow j} N_{i} ; i=0,1,2, \ldots, I_{\max }
$$

Results from the quantum mechanical solution of the harmonic oscillator are used to relate the 
various quantum transition rates to one another, and then the master equation may be summed over all quantum states to obtain the Landau-Teller equation:

$$
\frac{D \rho e_{v}}{D t}=\frac{\partial}{\partial x_{t}}\left(k_{v} \frac{\partial T_{v}}{\partial x_{i}}\right)+\rho \frac{e_{v}^{e q}\left(T_{t}\right)-e_{v}}{\tau_{L T}}
$$

An empirical expression (given below) is used to model the Landau-Teller relaxation time scale. First, the thermodynamic data for each species at every grid point are calculated using the following equations:

$$
\begin{aligned}
& \frac{C p}{R}=a_{1}+a_{2} T+a_{3} T^{2}+a_{4} T^{3}+a_{5} T^{4} \\
& \frac{h}{R T}=a_{1}+\frac{1}{2} a_{2} T+\frac{1}{3} a_{3} T^{2}+\frac{1}{4} a_{4} T^{3}+\frac{1}{5} a_{5} T^{4}+\frac{a_{6}}{T} \\
& \frac{s}{R}=a_{1} \ln T+a_{2} T+\frac{1}{2} a_{3} T^{2}+\frac{1}{3} a_{4} T^{3}+\frac{1}{4} a_{5} T^{4}+a_{7}
\end{aligned}
$$

and the Landau-Teller expression for the vibrational relaxation time is calculated using the 6coefficient specified.

$$
\tau_{L T}=\frac{b_{1} T^{b_{2}} \exp \left[\left(b_{3} / T\right)^{b_{4}}\right]}{p\left(1-b_{5} \exp \left(-b_{6} / T\right)\right)}
$$

where $b_{1}=7.1 E-4, b_{2}=0, b_{3}=1.91 E 6, b_{4}=0.3333, b_{5}=0, b_{6}=0$ are used here. To solve this vibrational energy equation, the source term is linearized to result in an explicit term and an 
implicit term. This treatment is important for an unconditionally stable solution of the equation.

That is,

$$
\begin{aligned}
& \text { Explicit source term }=\rho \frac{e_{v}^{e q}\left(T_{t}\right)}{\tau_{L T}} \\
& \text { Implicit source term }=-\frac{\rho}{\tau_{L T}} e_{v}
\end{aligned}
$$

The vibrational temperature is used to influence the reaction rates of chemical reactions by assuming that the rate coefficients for dissociation are functions of the geometrical mean temperature between $\mathrm{T}$ and $\mathrm{T}_{\mathrm{v}}{ }^{\mathrm{P} 1}$

\section{Non-Equilibrium Air Chemistry}

A general system of chemical reactions can be written in terms of its stoichiometric coefficients ( $v_{\mathrm{ij}}$ and $v_{\mathrm{ij}}$ ) ) and the $i^{\text {th }}$ chemical species name $\left(M_{i}\right)$ of the $j^{\text {th }}$ reaction as

$$
\sum_{i} v_{i j} M_{i}=\sum_{i} v_{i j}^{\prime} M_{i}
$$

The net rate of change in the molar concentration of species $i$ due to reactions $j, X_{i j}$, and the species production rate can be written as: 


$$
\begin{aligned}
& X_{i j}=\left(v_{i j}^{\prime}-v_{i j}\right)\left[K_{j j} \prod_{i}\left(\frac{\rho Y_{i}}{M_{w i}}\right)^{n_{i j}}-K_{b j} \prod_{i}\left(\frac{\rho Y_{i}}{M_{w i}}\right)^{v_{i j}}\right] \\
& \dot{\omega}_{i}=M_{w_{i}} \sum_{j} X_{i j}
\end{aligned}
$$

The forward (Arrhenius law) and backward reaction rates for each reaction is given by:

$$
\begin{aligned}
& K f_{l}=A_{J} T^{B_{i}} \operatorname{Exp}\left(-\frac{E_{1}}{R T}\right) \\
& K b_{\jmath}=\frac{K_{f_{1}}}{K e_{1}}
\end{aligned}
$$

where $K e_{j}$ is the equilibrium coefficient

$$
K_{e}=(R T) \sum_{i=1}^{-\sum_{i j}^{n}\left(v_{i j}^{\prime}-v_{i j}\right)} \operatorname{Exp}\left[\frac{\sum_{i=1}^{n}\left(v_{i j}^{\prime}-v_{i j}\right) g_{i}}{R T}\right]
$$

A point-implicit (operator splitting) method is employed to solve the chemistry system. For the breakdown of air, Park's multitemperature air chemistry ${ }^{\mathrm{Pl}}$ is based in this study. As shown in Appendix 1, this mechanism composes of the dissociation, NO exchange, associative ionization, charge exchange, electron-impact ionization, and radiative recombination reactions. With this mechanism, electrons are produced first by the associative ionization process and more electrons are produced by the electron-impact reactions. Since the number of electrons doubles in each such event, electron density increases exponentially, in the form of an avalanche. ${ }^{P 1}$ This mechanism provides the initial electron density for ignition. The combination of the avalanche 
of electrons (produced from this mechanism) and the absorption of incident laser energy drives the subsequent optical breakdown. It can be seen that IB is embedded in the dissociation, associative ionization, and electron-impact ionization reactions. The reaction rates were validated $^{\mathrm{PI}}$ with experiments of postshock temperatures ranging from $20,000-60,000 \mathrm{k}$ which are inline with the maximum computed breakdown temperatures. Notice heavy gas temperature $\mathrm{T}$ is used in place of $\mathrm{T}_{\mathrm{a}}\left(=\mathrm{T}^{\mathrm{T} / 2} \mathrm{~T}_{\mathrm{v}}{ }^{1 / 2}\right)$ for dissociation reactions in current study.

\section{$\underline{\text { Plasma Initiation }}$}

Understanding the mechanisms responsible for plasma initiation (ignition) has been the subject of ongoing research. A spark gap, an extremely intense pulse of laser energy striking the focal point, seed molecules or particles, or a retractable tungsten target placed at the focal point ${ }^{\mathrm{E} 2}$ have been used as the source of free electrons for initiation. ${ }^{\mathrm{Gl}}$ It has also been shown that plasma can be ignited quite easily off metal surfaces. ${ }^{\mathrm{G} 1}$ Likewise in numerical modeling, initial free electrons are required for plasma ignition. This means determining an initial electron density or a threshold breakdown intensity. For example, Ergun ${ }^{\mathrm{E} 2}$ described an initial electron density is necessary for modeling the ignition of a hydrogen plasma initiated with a retractable tungsten wire. In this study, a threshold breakdown intensity approach is devised in anticipation that different threshold breakdown intensity may be encountered for different surface material, target approach, and laser power. The advantage of this approach is that threshold breakdown intensity can either be calibrated or measured. For example, the effect of seeds on laser breakdown intensity was reported. ${ }^{\mathrm{G} 1}$ This is accomplished by performing parametric studies on plasma initiation with spark time, spark area, and spark power. A "spark" provides a fixed amount of 
energy (spark energy) in an area (spark area) centered around the focal point for a fixed amount of time (spark time). When the strength of a spark reaches a threshold, or the threshold breakdown intensity is satisfied, a laser supported combustion (LSC) or detonation (LSD) is initiated.

That means enough seed electrons are produced through the associative ionization reactions and the subsequent optical breakdown (ignition) is thereby possible. It is found that an ignition is sustainable when the energy absorbed by plasma reaches $15 \%$ of that of the delivered. Spark times computed based on that criterion are ranging from $0.4 \mu$ s to $1.2 \mu \mathrm{s}$. As expected, lower laser pulse energies require longer spark times for ignition. It is also reasonable to assume that the plasma initiates in an (spark) area enclosed by the two outer laser rays and an arc with an origin at the focal point. This is a cone (in a two-dimensional sense) and the (spark) radius is determined empirically as $1 \mathrm{~mm}$. Finally, the spark power is also empirically determined as $15 \%$ of that of the incident laser - its theoretical maximum.

\section{Laser Radiation}

Geometric optics is used to simulate the local intensity of the laser beam, which is split into a number of individual rays. In the presence of absorption, the local intensity of each ray follows the Beer's law:

$$
\frac{d I_{i}}{d s_{i}}=-\kappa_{l} I_{i}
$$

Electron is the only plasma species that absorbs the laser energy. The absorption coefficient of 
the $\mathrm{CO}_{2}$ laser radiation, corrected for stimulated emission in the single ionization range, is approximated by the formula: ${ }^{R I}$

$$
\kappa_{l \mathrm{CO}_{2}}=\frac{5.72 p_{e}{ }^{2} \ln \left[27\left(T / 10^{4}\right)^{4 / 3} p_{e}{ }^{-1 / 3}\right]}{\left(T / 10^{4}\right)^{7 / 2}}
$$

A ray may change its propagating direction due to the inhomogeneous refractive index within the hot plasma. The index of refraction is taken from Edwards and Pleck: ${ }^{\text {El }}$

$$
n=\left[1-\left(\frac{n_{e} \lambda_{l} q_{e} k_{c}}{m_{e} c^{2} \pi}\right)\right]^{1 / 2}
$$

The refracted angle is associated with the refractive index through the Snell's law: ${ }^{\mathrm{M} 3}$

$$
n_{1} \sin \theta_{1}=n_{2} \sin \theta_{2}
$$

where $n_{1}$ and $n_{2}$ represent the refractive indexes for two different control volumes, and $\theta_{1}$ and $\theta_{2}$ are the incident and refracted directions with respect to the normal direction of the interface between two volumes. 


\section{Non-equilibrium Plasma Radiation}

Treatment of radiative heat transfer of plasma is different from that of laser and the solution of the radiative transfer equation (RTE) is required. Neglecting transients and assuming a noscattering medium, the complete RTE becomes

$$
\frac{d I(s, \vec{\Omega})}{d s}+\kappa I(s, \vec{\Omega})=j(s)
$$

In equilibrium gases, the electronic energy-level populations are determined as a function of a uniquely defined equilibrium temperature according to a Boltzmann distribution, and $\kappa$ and $j$ are related according to Kirchhoff's law as $j(s)=\kappa I^{b}(s)$, where $I^{b}(s)$ is blackbody intensity determined by Planck function. In non-equilibrium flow, however, all these simple relations no longer apply, whereas the non-equilibrium absorption and emission coefficients must be determined by the non-equilibrium populations of each energy level and transitions among various energy levels.

Air plasma includes following atomic and molecular species: $\mathrm{O}, \mathrm{N}, \mathrm{NO}, \mathrm{N}^{+}, \mathrm{O}^{+}, \mathrm{NO}^{+}, \mathrm{N}_{2}$, $\mathrm{O}_{2}, \mathrm{~N}_{2}{ }^{+}, \mathrm{O}_{2}{ }^{+}$, and $\mathrm{e}^{-}$which all contribute to the non-equilibrium radiative heat transfer. To determine $\mathrm{\kappa}$ and $j$ for these species, the following four radiative transitions must be taken into account: atomic line transitions, atomic bound-free transitions, atomic free-free transitions, and molecular transitions. Currently, there are two codes available which provide detailed information on these transitions. One is NEQAIR developed by Park $^{\mathrm{P} 2}$ and another is LORAN

developed by Hartung. ${ }^{\mathrm{H} 1}$ Because NEQAIR code involves large database, they are rarely applied 
in practical problems. In this study, LORAN code is used to calculate the air plasma radiative properties.

With the determination of $\kappa$ and $j$, the RTE can be solved by either deterministic or stochastic approach. Unlike the computational plasma aerodynamics governing equations, RTE is an integral differential equation and numerical treatment is, thus, different from those for differential equations. Currently, there are several methods available for solving non-equilibrium radiative heat transfer which include Monte Carlo method, P-1 method, quadromoment method, discrete ordinates method (DOM), discrete transfer method (DTM), etc. The Monte Carlo is accurate but too time-consuming for practical applications. The P-1 and quadromoment methods are only accurate for optically thick medium. The DOM and DTM are mathematically simple and can provide accurate results for all optical ranges if the discrete direction number is reasonable large. Thus, the DOM and DTM are very suitable for modeling radiation with a participating medium. For the laser propelled vehicle application, the high temperature region where nonequilibrium radiation is prominent is usually relatively small compared to the flow region considered. If the DOM is applied, RTE must be solved in the entire flow region, thus, much of the computational time is wasted in the regions where radiation is not important. However, with the use of the DTM, RTE can be solved in a designated region. Therefore, the efficient DTM is selected to solve the RTE. Detailed numerics of DTM is described in Ref. C1.

\section{Plasma Resonance}

The plasma frequency is described as a property of a space-charge-neutral plasma ${ }^{\mathrm{B} 1, \mathrm{H} 2}$ by which the motion of the electrons in specific electrostatic oscillations is characterized. The 
plasma frequency $\omega_{\mathrm{p}}$ of the electrostatic oscillation of the electrons is defined as ${ }^{\mathrm{BI}}$

$$
\omega_{\mathrm{p}}^{2}=\mathrm{n}_{\mathrm{e}} \mathrm{q}_{\mathrm{e}}^{2} / \mathrm{me}_{\mathrm{e}} \varepsilon_{0}
$$

It is seen that plasma frequency is a function only of the electron density. The electrostatic oscillations cause a resonance of the plasma to incident (laser) electromagnetic waves of the same frequency which are then totally reflected. ${ }^{\mathrm{H} 2}$ A critical electron density above which the laser beam is totally reflected can then be obtained by equating the frequency of the incident laser with that of the plasma.

Once the local electron density reaches the critical value, a "total reflection" condition has to be applied to the incident beam since the plasma becomes "opaque" and no beam can penetrate through the plasma front. This is achieved by assuming a total absorption at the plasma front and then a redistribution of the energy. $\mathrm{Zhu}^{\mathrm{Zl}}$ noted that during the critical plasma resonance, the incident laser energy is to exciting the electron to a higher energy level, to reflecting and radiating diffusely, and to a lesser extent, escaping in the form of light by returning the excited electron to a lower energy level. The first energy redistribution mechanism can be viewed as an energy conversion, whereas the second and third ones can be grouped as an energy loss. Although the percentage of each is not theoretically known, an energy conversion percentage during the critical plasma resonance can be calibrated with the test results. In this study, a $30 \%$ energy conversion is used.

\section{Experimental Setup}


The $10 \mathrm{~kW}$ Pulsed Laser Vulnerability Test System (PLVTS) $\mathrm{CO}_{2}$ laser was used to provide the beam energy for Laser Lightcraft thrust experiments. The $\mathrm{CO}_{2}$ laser delivered up to $400 \mathrm{~J}$ single-pulses at a pulse width of $18 \mu \mathrm{s}$. Several variations of the basic Laser Lightcraft design (Model 200 series) similar to those described in Ref. M2 were examined and the test results of the bare aluminum Model \#200-3/4 vehicle is chosen for this study. The impulse measurements were conducted with a pendulum apparatus. This technique employed a velocimeter coil, which was used in previous work to determine the impulse imparted to a flat plate using the PHAROS III laser at Naval Research Laboratory. ${ }^{\text {L2 }}$ The Laser Lightcraft was suspended and suitably weighed before being subjected to a single pulse of energy from the PLVTS laser. The impulse hammer had been calibrated with clay balls and the data agree with each other well. Details of the laser, measurement technique, and pendulum apparatus can be found in Refs. M1 and M2.

\section{Computational Grid Generation}

Figure 1 shows the layout of a computational grid. As described in Refs. M1 and M2, the Laser Light vehicle consists of a forebody (nose), an annular shroud, and a PDW engine (parabolic optic). Only half of the grid shown in Fig. 1 is actually solved due to the axisymmetric formulation. An eight-zone structured grid was generated first using a grid generation code UMESH. ${ }^{\mathrm{C} 2}$ The grid is then fed to an auxiliary program such that a one-zone unstructured-grid is resulted for the actual computation. Quadrilateral elements are used such that wall boundary layer can be computed if needed. High grid density is used in the inner shroud region for capturing the optical breakdown and pulsed detonation wave processes. 


\section{$\underline{\text { Results and Discussion }}$}

A series of computations have been performed for average laser pulse energies of 75,100 , $150,200,300$ and $400 \mathrm{~J}$. Maximum computed temperatures ranging from 51,000 to $60,000 \mathrm{~K}$ fall within the computed post-shock temperatures of 20,000 to $60,000 \mathrm{~K}$ using Park's multitemperature air chemistry. ${ }^{\mathrm{P} 1}$ Laser supported detonation occurs in all cases with maximum Mach number reaching 1.6. Laser supported combustion then ensues as the plasma front expands, whereas the Mach number decreases to subsonic level. Figure 2 shows the computed temperature contours and laser beam traces at an elapse time of $0.5 \mu \mathrm{s}$ and average laser pulse energy of $400 \mathrm{~J}$. It can be seen that the laser beam reflects specularly on the optical surface and focuses onto a focal "point" on the shroud where the breakdown of air starts. Ten laser rays are plotted for clarity, whereas two hundred rays are actually used in the computation.

It is probably best to recount the phenomenon of optical breakdown, also known as the optical detonation, at this moment. This was put forward by Raizer ${ }^{\mathrm{R} 1}$ in detail in his book. Essentially, a strong shock wave is generated in the region where laser radiation is absorbed by the plasma and heat is deposited very rapidly. This shock wave travels through the medium, heating and ionizing such that the medium becomes capable of absorbing more laser radiation. The laser energy is deposited in successive layers of a medium adjoining the front of the shock wave subjecting to the laser radiation. These layers themselves become energy sources which maintain the shock wave. Hence, the shock wave moves along the optical channel opposite to the laser beam, whereas during the initial stages it is maintain by the beam and does not decay.

Figure 3 shows the temperature contours at an elapse time of $10 \mu$ s where the optical breakdown is being fed by the laser energy and the plasma front grows. Notice the rays are 
allowed to bend as the index of refraction varies with the expanding plasma. As the local electron density of a plasma front reaches the critical value such that plasma resonance occurs, the local plasma becomes opaque and the regular laser energy absorption moves to other area of the plasma front. This process is nonlinear and may explain the irregular shape of the plasma front. It is anticipated that laser beam totally reflects if the plasma resonance spreads to the entire plasma front. The phenomenon of plasma resonance disappears as the plasma front expands continuously, while the electron density decreases continuously. The computed optical breakdown phenomenon appears agreeing with that described by Raizer. ${ }^{\text {RI }}$ Notice the spreading of the shock wave outside the optical channel results in gradual attenuation because there is no energy to maintain it. In the downbeam direction the shock wave is either weakly maintained by the laser radiation than in the upbeam direction, or is not maintained by the laser radiation during plasma resonance.

Figure 3 shows the temperature contours at an elapse time of $20 \mu \mathrm{s}$. At this time, the laser beam is turned off since the pulse width is fixed at $18 \mu \mathrm{s}$, thus the shock wave is not being maintained by the laser radiation. The computational results show that a pulsed detonation wave usually takes place at approximately 10 to $20 \mu$ s after start command. The higher the pulse energy, the earlier the generation of a pulsed detonation wave. Part of this propagating detonation wave will hit the parabolic optic and reflect off the surface. The reflected and nonreflected portions would combine to make a propagating Mach stem. It should be noted that the phenomenon of a propagating Mach stem on the optical surface is very similar to that of a propagating Mach disc inside a bell nozzle during the start-up transient. ${ }^{\text {Wl, } 2}$ This is because the aerospike optic geometry is an "inside-out" version to that of a contoured bell nozzle. 
As the Mach stem moves past the end of shroud, the part that is attached to the inner-shroud starts to wrap around the outer-shroud. As the wrap around progresses, the shock wave weakens and grows like an ellipsoid. A forebody attaching "leg" would develop, as shown in the pressure contours of Fig. 5. Eventually, the "leg" leaves the nose and the ellipsoid expands to infinity. That completes the propulsion physics of the Laser Lightcraft for a single pulse of laser energy.

Coupling coefficient is the generated thrust divided by the delivered average laser pulse energy. It is a performance measure particular to the pulsed laser propulsion at the air-breathing mode, and conceptually similar to the specific impulse (generated thrust divided by mass flow rate) of a chemical rocket. Figure 6 shows a comparison of the coupling coefficients between the model prediction and test measurement. The model predicted coupling coefficients agreed reasonably well with those of the measurement. At this writing, the effect of non-equilibrium radiation has not been added to the result, hence there is room for improvement once it is considered.

\section{Conclusion}

A computational plasma aerodynamics model has been developed to study the propulsion physics of an experimental Laser Lightcraft. The model development is based on a build block approach such that the model can be improved continuously with improved understanding of the physics. The model predicted laser propulsion physics such as the optical breakdown and detonation wave propagation agree well with those described in the literature. The model predicted coupling coefficient for a Model \#200 Laser Lightcraft agreed reasonably well with those of the measurement. 


\section{Acknowledgment}

The authors wish to thank Sandy Kirkindall of Laser Propulsion and John Cole of Advanced

Propulsion for supporting this study. The lead author wishes to thank Chris Beairsto of the Directed Energy Team for the laser specifics and James Reilly of NorthEast Science and Technology for discussions on plasma physics.

\section{References}

B1. Brown, Sanborn C., Basic Data of Plasma Physics, The Technology Press of The Massachusetts Institute of Technology and John Wiley \& Sons, Inc., New York.

B2. Brandstein, A., and Levy, Y., "Laser Propulsion System for Space Vehicles," Journal of Propulsion and Power, Vol. 14, No. 2, 1998, pp. 261-269.

B3. Barth, T.J., "Recent Developments in High Order K-Exact Reconstruction on Unstructured Meshes,' AIAA Paper 93-0668, 1993.

C1. Coelho, P. J. and Carvalho, M. G., "A Conservative Formulation of the Discrete Transfer Method," Journal of Heat Transfer, Vol. 119, No. 1, 1997, pp. 118-128.

C2. Chen, Y.S., "UNIC-MESH: A Model Builder \& Mesh Generator, Graphical User Interface," Engineering Sciences, Inc. Internal Report, UNIC-Mesh-V.3.0, 2000.

E1. Edwards, A. L. and Fleck, Jr. J. A., "Two-dimensional Modeling of Aerosol-Induced Breakdown," Journal of Applied Physics., Vol. 50, No. 6, 1979, pp. 4307-4313.

E2. Ergun, M.A., Modeling and Experimental Measurements of Laser Sustained Hydrogen Plasmas, Ph.D. Dissertation, University of Illinois at Urbana-Champaign, 1993. 
F1. Fletcher, R., "Conjugate Gradient Methods for Indefinite Systems," Lecture Notes in Mathematics, Vol. 506, 1976, pp. 73-89.

G1. Glumb, R.J., and Krier, H., "Concepts and Status of Laser-Supported Rocket Propulsion," Journal of Spacecraft and Rockets, Vol. 21, NO. 1, 1984, pp. 70-79.

H1. Hartung, Lin, “Theory and User's Manual for Loran Code,” NASA TM-4564, 1994.

H2. Hora, Heinrich, Laser Plasmas and Nuclear Energy, Plenum Press, New York and London, 1975.

J1. Jeng, San-Mou, and Keefer, Dennis, "Theoretical Evalualtion of Laser-Sustained Plasma Thrister Performance," J. Propulsion, Vol. 5, No. 5, Sept-Oct., 1989, pp. 577-581.

K1. Kantrowitz, A., "Propulsion to Orbit by Ground-Based lasers," Astronautics and Aeronautics, Vo.. 10, No. 5, May 1972, pp. 74-76.

K2. Kemp, N.H., and Root, R.G., "Analytical Study of LSC Waves in Hydrogen," Journal of Energy, Vol. 3, Jan-Feb, 1979, pp. 40-49.

L1. Landau, L., and Teller, E., "Zur Theorie der Schalldispersion," Physic Z. Sowjetunion, Vol. 10, No. 34, 1936.

L2. Lyons, P.W., Myrabo, L.N., Jones, R.A., Nagamatsu, H.T., and Manka, C., "Experimental Investigation of a Unique Airbreathing Pulsed Laser Propulsion Concept," AIAA Paper 91-1922, 1991.

M1. Myrabo, L.N., Messitt, D.G., and Mead, F.B., Jr., "Ground and Flight Tests of a Laser Propelled Vehicle," AIAA Paper 98-1001, Jan., 1998.

M2. Mead, F.B., Jr., and Myrabo, L.N., Messitt, D.G., "Flight and Ground Tests of a LaserBoosted Vehicle," AIAA Paper 98-3735, July, 1998. 
M3. Modest, M. F., "Radiative Heat Transfer," McGraw-Hill, NY, 1993.

M4. Molvik, G.A., Choi, D., and Merkle, C.L., "A Two-Dimensional Analysis of Laser Heat Adition in a Constant Absorptivity Gas," AIAA Journal, Vol. 23, No. 7, 1985, pp. 1053-1060.

P1. Park, C., "Review of Chemical-Kinetic Problems of Future NASA Missions, I: Earth Entries," Journal of Thermophysics and Heat Transfer, Vol. 7, No. 3, 1993, pp. 385-398.

P2. Park, C., "Nonequilibrium Air Radiation Program: User's Manual," NASA TM-86707, 1985. P3. Park, C., Nonequilibrium Hypersonic Aerothermodynamics, Wiley, New York, 1990.

R1. Raizer, Y.P., and Tybulewicz, A., "Laser-Induced Discharge Phenomena", Studies in Soviet Science, Edited by Vlases, G.C., and Pietrzyk, Z.A., Consultants Bureau, New York, 1977.

R2. Raizer, Y.P., "Subsonic Propagation of a Light Spark and Threshold Conditions for the Maintenance of Plasma by Radiation," Soviet Physics JETP, Vol. 31, Dec., 1970, pp. 1148-1154.

S1. Sharma, S.P., Gillespie, W.D., and Meyer, S.A., "Shock Front Radiation Measurements in Air," AIAA Paper 91-0573, Jan. 1991.

S2. Saad, Y., and Schultz, M.H., "GMRES: A Generalized Minimal Residual Algorithm for Solving Nonsymmetric Linear Systems," SIAM Journal of Scientific and Statistical Computing.

Vol. 7, No. 3, Jan. 1986, pp. 856-869.

W1. Wang, T.-S., "Numerical Study of the Transient Nozzle Flow Separation of Liquid Rocket Engines," Computational Fluid Dynamics Journal, Vol.1, No.3, Oct. 1992, pp. 319-328.

W2. Wang, T.-S. and Chen Y.-S., "Unified Navier-Stokes Flowfield and Performance Analysis of Liquid Rocket Engines," Journal of Propulsion and Power, Vol. 9, No. 5, Sept.-Oct. 1993, pp.678-685.

Z1. Zhu, S., USRA, MSFC, personal commumication. 
Appendix 1. Air breakdown kinetics mechanism.

\begin{tabular}{|c|c|c|c|c|c|c|}
\hline Reaction & $\mathrm{M}^{*}$ & $\mathrm{~T}_{\mathrm{x}}^{*}$ & $\mathrm{~A}$ & $\mathrm{~B}$ & $E / R$ & Ref. \\
\hline \multicolumn{7}{|l|}{ Dissociation Reactions } \\
\hline \multirow[t]{11}{*}{$\mathrm{N}_{2}+\mathrm{M}=\mathrm{N}+\mathrm{N}+\mathrm{M}$} & $\mathrm{N}$ & $T_{a}$ & $3.0^{22}$ & -1.60 & 113,200 & P3 \\
\hline & $\mathrm{O}$ & & $3.0^{22}$ & & & \\
\hline & $\mathrm{N}_{2}$ & & $7.0^{21}$ & & & \\
\hline & $\mathrm{O}_{2}$ & & $7.0^{21}$ & & & \\
\hline & NO & & $7.0^{21}$ & & & \\
\hline & $\mathrm{N}^{+}$ & & $3.0^{22}$ & & & \\
\hline & $\mathrm{O}^{+}$ & & $3.0^{22}$ & & & \\
\hline & $\mathrm{N}_{2}^{+}$ & & $7.0^{21}$ & & & \\
\hline & $\mathrm{O}_{2}^{+}$ & & $7.0^{21}$ & & & \\
\hline & $\mathrm{NO}^{+}$ & & $7.0^{21}$ & & & \\
\hline & $\mathrm{e}^{-}$ & & $1.2^{25}$ & & & S1 \\
\hline \multirow[t]{10}{*}{$\mathrm{O}_{2}+\mathrm{M}=\mathrm{O}+\mathrm{O}+\mathrm{M}$} & $\mathrm{N}$ & $\mathrm{T}_{3}$ & $1.0^{22}$ & -1.50 & 59,500 & P3 \\
\hline & $\mathrm{O}$ & & $1.0^{22}$ & & & \\
\hline & $\mathrm{N}_{2}$ & & $2.0^{21}$ & & & \\
\hline & $\mathrm{O}_{2}$ & & $2.0^{21}$ & & & \\
\hline & NO & & $2.0^{21}$ & & & \\
\hline & $\mathrm{N}^{+}$ & & $1.0^{22}$ & & & \\
\hline & $\mathrm{O}^{+}$ & & $1.0^{22}$ & & & \\
\hline & $\mathrm{N}_{2}^{+}$ & & $2.0^{21}$ & & & \\
\hline & $\mathrm{O}_{2}^{+}$ & & $2.0^{21}$ & & & \\
\hline & $\mathrm{NO}^{+}$ & & $2.0^{21}$ & & & \\
\hline \multirow[t]{10}{*}{$\mathrm{NO}+\mathrm{M}=\mathrm{O}+\mathrm{O}+\mathrm{M}$} & $N$ & $\mathrm{~T}_{3}$ & $1.1^{17}$ & 0.00 & 75,500 & P3 \\
\hline & $\mathrm{O}$ & & $1.1^{17}$ & & & \\
\hline & $\mathrm{N}_{2}$ & & $5.0^{15}$ & & & \\
\hline & $\mathrm{O}_{2}$ & & $5.0^{15}$ & & & \\
\hline & NO & & $1.1^{17}$ & & & \\
\hline & $\mathrm{N}^{+}$ & & $1.1^{17}$ & & & \\
\hline & $\mathrm{O}^{+}$ & & $1.1^{17}$ & & & \\
\hline & $\mathrm{N}_{2}^{+}$ & & $5.0^{15}$ & & & \\
\hline & $\mathrm{O}_{2}^{+}$ & & $5.0^{15}$ & & & \\
\hline & $\mathrm{NO}^{+}$ & & $5.0^{15}$ & & & \\
\hline \multicolumn{7}{|l|}{ NO Exchange Reactions } \\
\hline $\mathrm{NO}+\mathrm{O}=\mathrm{N}+\mathrm{NO}$ & & $\mathrm{T}$ & $8.4^{12}$ & 0.00 & 19,450 & P3 \\
\hline $\mathrm{N}_{2}+\mathrm{O}=\mathrm{NO}+\mathrm{N}$ & & $T$ & $6.4^{17}$ & -1.00 & 38,400 & P3 \\
\hline \multicolumn{7}{|l|}{ Associative Ionization } \\
\hline $\mathrm{N}+\mathrm{O}=\mathrm{NO}^{+}+\mathrm{e}^{-}$ & & $\mathrm{T}$ & $8.8^{8}$ & 1.00 & 31.900 & P1 \\
\hline $\mathrm{O}+\mathrm{O}=\mathrm{O}_{2}^{+}+\mathrm{e}^{-}$ & & $\mathrm{T}$ & $7.1^{2}$ & 2.70 & 80.600 & P1 \\
\hline $\mathrm{N}+\mathrm{N}=\mathrm{N}_{2}^{+}+\mathrm{e}^{-}$ & & $\mathrm{T}$ & $4.4^{7}$ & 1.50 & 67,500 & $\mathrm{P} 1$ \\
\hline \multicolumn{7}{|l|}{ Charge Exchange } \\
\hline $\mathrm{NO}^{+}+\mathrm{O}=\mathrm{N}^{+}+\mathrm{O}_{2}$ & & $\mathrm{~T}$ & $1.0^{12}$ & 0.50 & 77,200 & $\mathrm{P} 3$ \\
\hline $\mathrm{N}^{+}+\mathrm{N}_{2}=\mathrm{N}_{2}^{+}+\mathrm{N}$ & & $\mathrm{T}$ & $1.0^{12}$ & 0.50 & 12,200 & P3 \\
\hline $\mathrm{O}_{2}^{+}+\mathrm{N}=\mathrm{N}^{+}+\mathrm{O}_{2}$ & & $\mathrm{~T}$ & $8.7^{13}$ & 0.14 & 28,600 & P3 \\
\hline $\mathrm{O}^{+}+\mathrm{NO}=\mathrm{N}^{+}+\mathrm{O}_{2}$ & & $\mathrm{~T}$ & $1.4^{5}$ & 1.90 & 26,600 & P3 \\
\hline $\mathrm{O}_{2}^{+}+\mathrm{N}_{2}=\mathrm{N}_{2}^{+}+\mathrm{O}_{2}$ & & $\mathrm{~T}$ & $9.9^{12}$ & 0.00 & 40,700 & $\mathrm{P} 3$ \\
\hline $\mathrm{O}_{2}^{+}+\mathrm{O}=\mathrm{O}^{+}+\mathrm{O}_{2}$ & & $\mathrm{~T}$ & $4.0^{12}$ & -0.09 & 18,000 & P3 \\
\hline $\mathrm{NO}^{+}+\mathrm{N}=\mathrm{O}^{+}+\mathrm{N}_{2}$ & & $\mathrm{~T}$ & $3.4^{13}$ & -1.08 & 12,800 & P3 \\
\hline $\mathrm{NO}^{+}+\mathrm{O}_{2}=\mathrm{O}_{2}^{+}+\mathrm{NO}$ & & $T$ & $2.4^{13}$ & 0.41 & 32,600 & P3 \\
\hline $\mathrm{NO}^{+}+\mathrm{O}=\mathrm{O}_{2}^{+}+\mathrm{N}$ & & $\mathrm{T}$ & $7.2^{12}$ & 0.29 & 48,600 & P3 \\
\hline $\mathrm{O}^{+}+\mathrm{N}_{2}=\mathrm{N}_{2}^{+}+\mathrm{O}$ & & $\mathrm{T}$ & $9.1^{11}$ & 0.36 & 22,800 & P3 \\
\hline $\mathrm{NO}^{+}+\mathrm{N}=\mathrm{N}_{2}^{+}+\mathrm{O}$ & & $\mathrm{T}$ & $7.2^{13}$ & 0.00 & 35,500 & P3 \\
\hline \multicolumn{7}{|l|}{ Electron-Impact Ionization } \\
\hline $\mathrm{O}+\mathrm{e}^{-}=\mathrm{O}^{+}+\mathrm{e}^{-}+\mathrm{e}^{-}$ & & $T_{e}$ & $3.9^{33}$ & -3.78 & 158,500 & P3 \\
\hline $\mathrm{N}+\mathrm{e}^{-}=\mathrm{N}^{+}+\mathrm{e}^{-}+\mathrm{e}^{-}$ & & $\mathrm{T}_{\mathrm{e}}$ & $2.5^{34}$ & -3.82 & 168,600 & P3 \\
\hline \multicolumn{7}{|l|}{ Radiative recombination } \\
\hline $\mathrm{O}^{+}+\mathrm{e}^{-}=\mathrm{O}$ & & $\mathrm{T}_{\mathrm{e}}$ & $1.07^{11}$ & -0.52 & & $\mathrm{Pl}$ \\
\hline $\mathrm{N}^{+}+\mathrm{e}^{-}=\mathrm{N}$ & & $\mathrm{T}_{e}$ & $1.52^{11}$ & -0.48 & & $\mathrm{Pl}$ \\
\hline
\end{tabular}

* $\mathrm{M}$ stands for third-body collision partner, $\mathrm{T}_{\mathrm{x}}$ is the controlling temperature. 


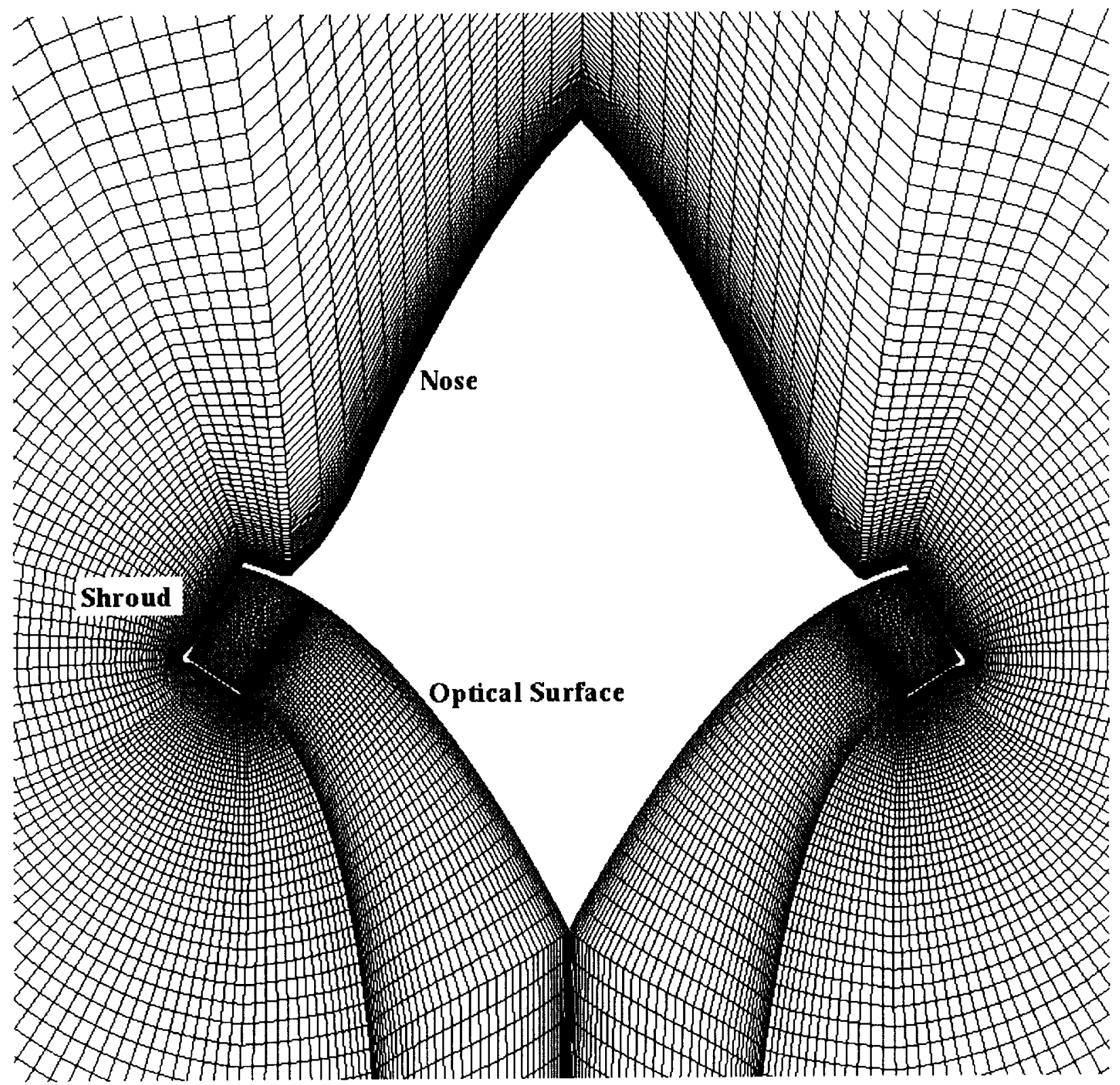

Fig. 1 The layout of a computational grid for the laser lightcraft. 


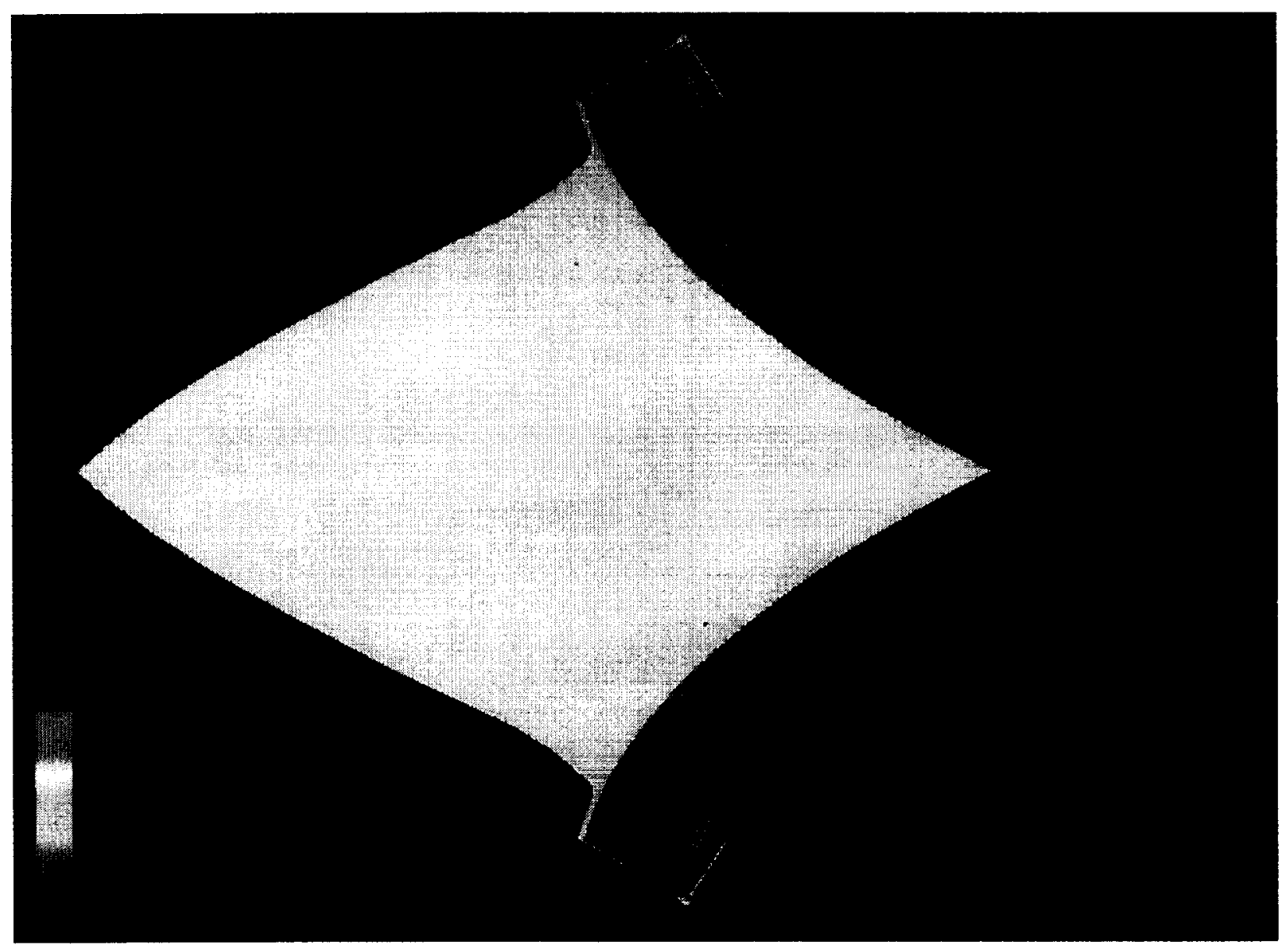

Fig. 2 Computed temperature contours and laser ray traces at $0.5 \mu$ s. 


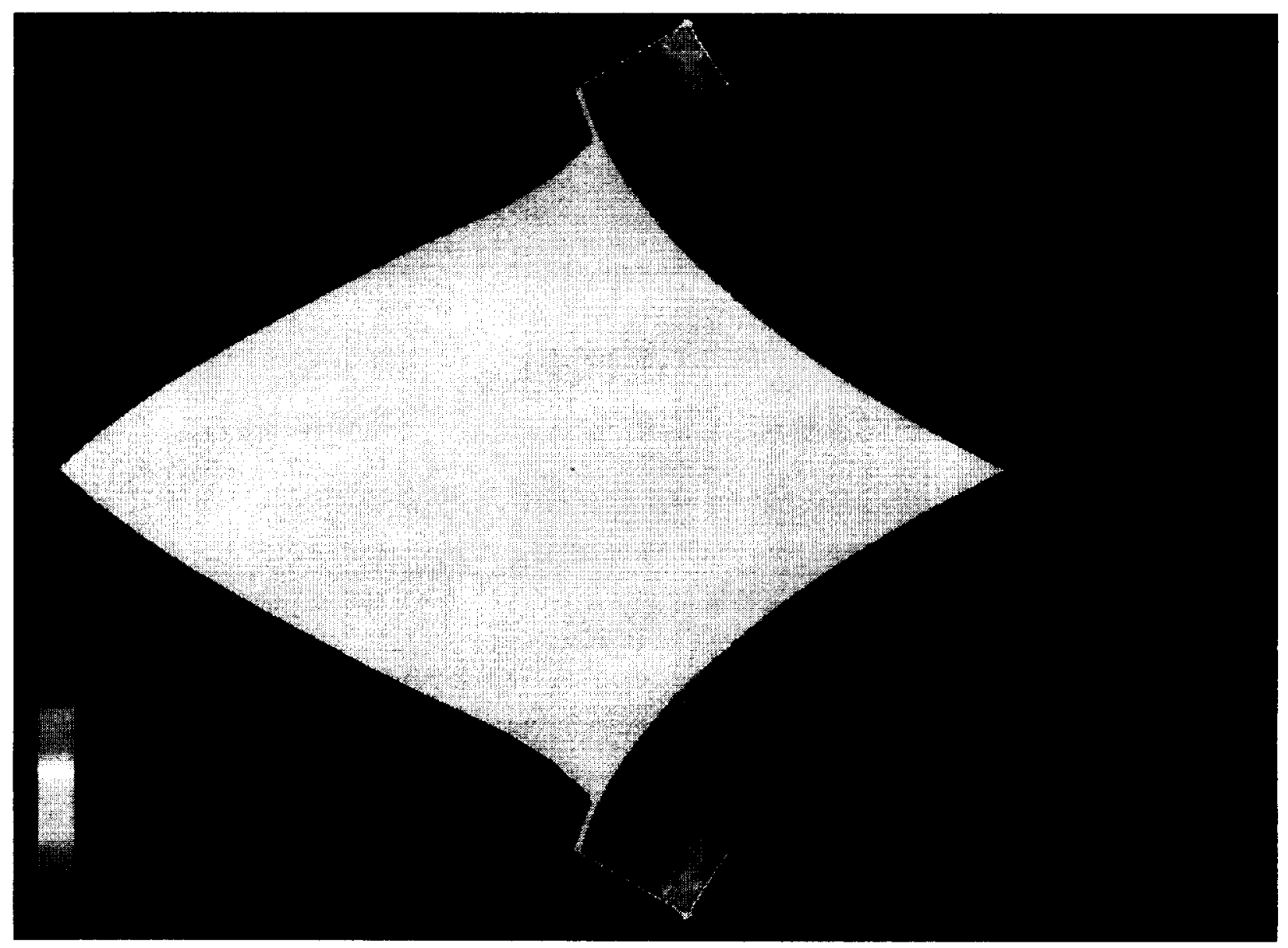

Fig. 3 Computed temperature contours and laser ray traces at $10 \mu \mathrm{s}$. 


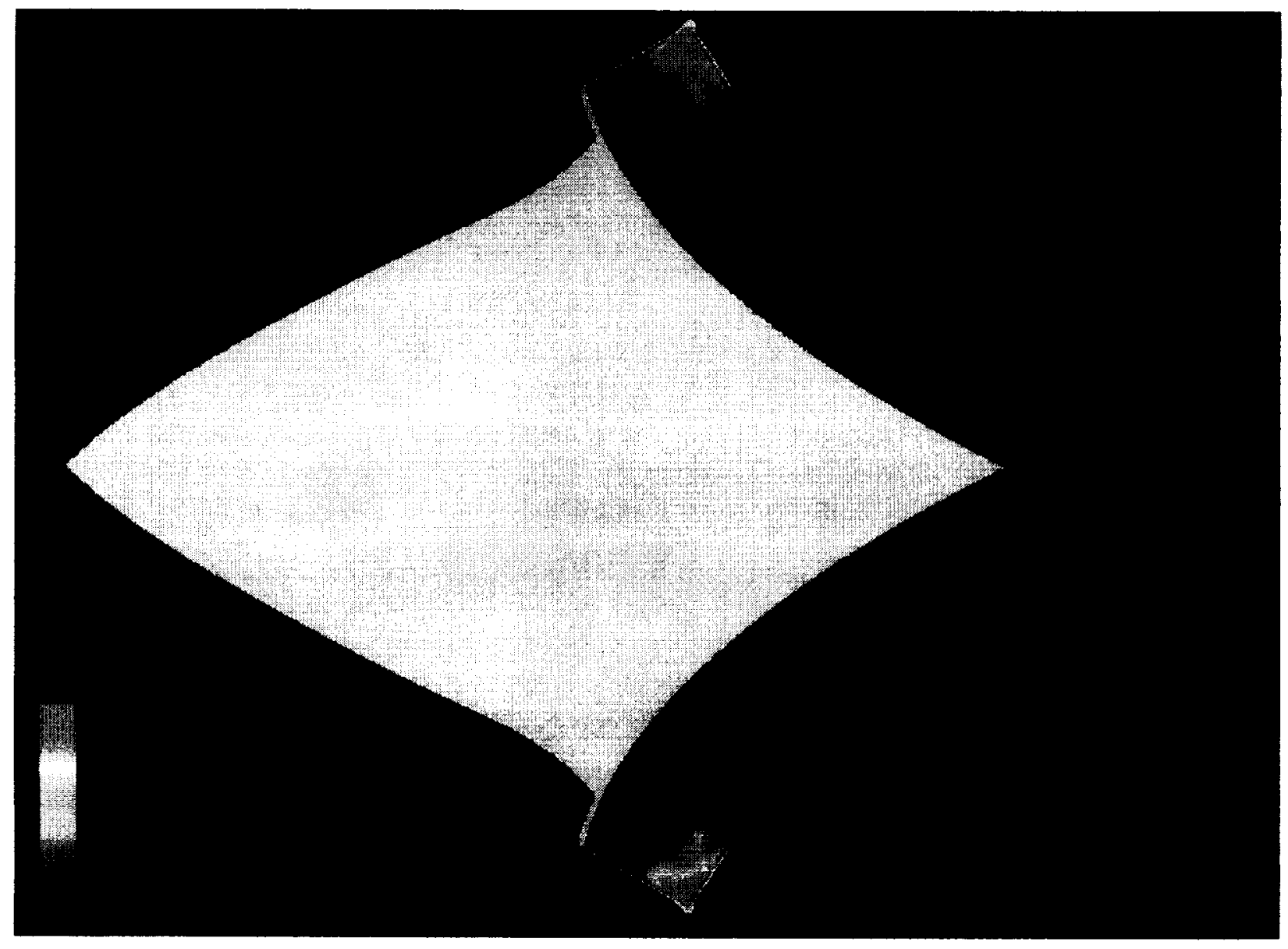

Fig. 4 Computed temperature contours at $20 \mu \mathrm{s}$. 


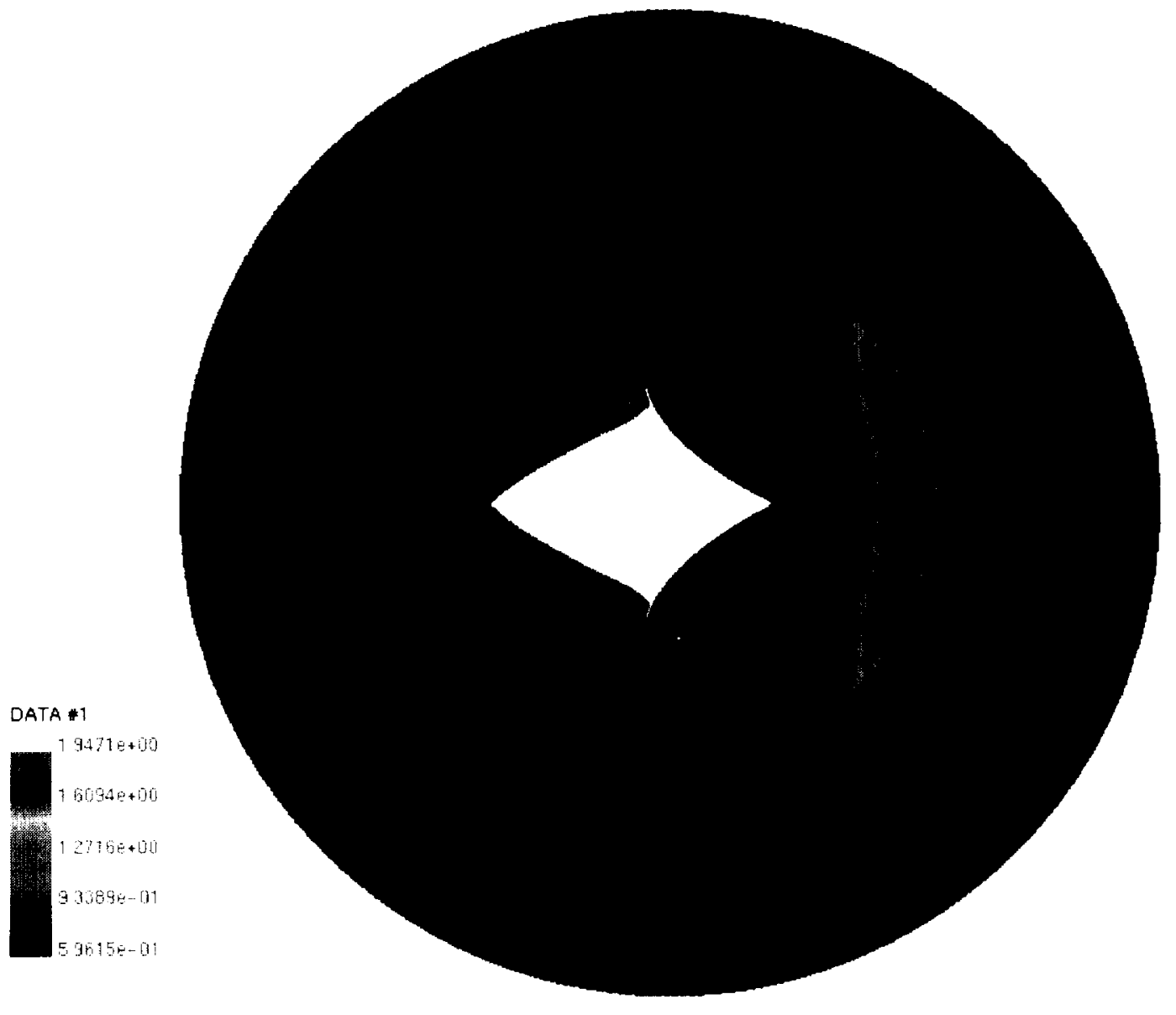

Fig. 5 Computed pressure contours at $105 \mu \mathrm{s}$. 


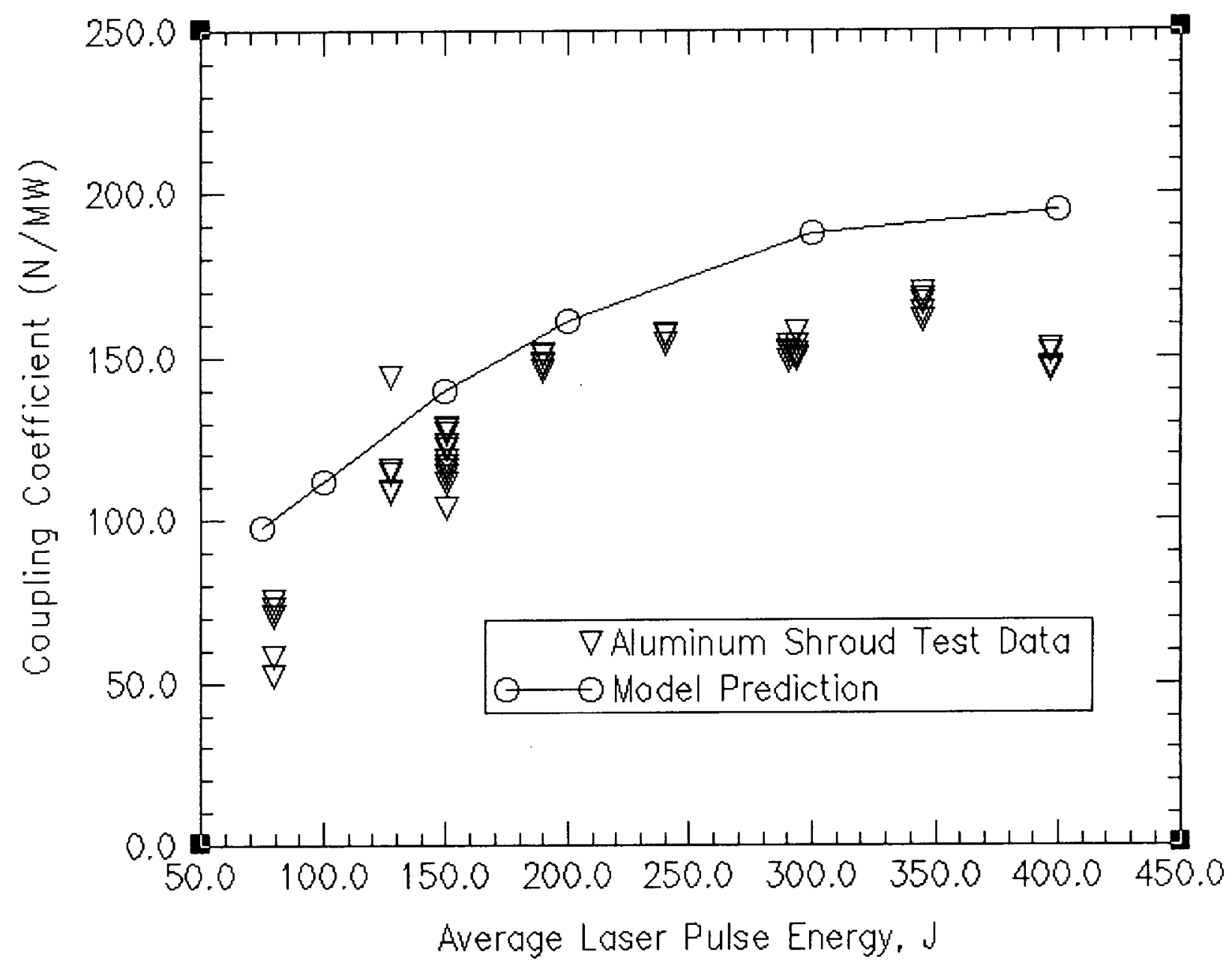

Fig. 6 A comparison of the coupling coefficients. 